\title{
STUDY ON CLASSIFICATION ACCURACY INSPECTION OF LAND COVER DATA AIDED BY AUTOMATIC IMAGE CHANGE DETECTION TECHNOLOGY
}

\author{
Wen-jun XIE ${ }^{1, *}$, Li ZHANG ${ }^{1}$, Hai-peng CHEN $^{1}$, Jin ZHOU ${ }^{1}$, Wen-juan MAO ${ }^{1}$ \\ ${ }^{1}$ National Quality Inspection and Testing Center for Surveying and Mapping Products, Beijing, China - xwj_13@126.com
}

Commission III, ICWG III/IVb

KEY WORDS: National Geographic Conditions Monitoring, Land Cover Data, Quality Inspection, Classification Accuracy, Change Detection

\begin{abstract}
:
The purpose of carrying out national geographic conditions monitoring is to obtain information of surface changes caused by human social and economic activities, so that the geographic information can be used to offer better services for the government, enterprise and public. Land cover data contains detailed geographic conditions information, thus has been listed as one of the important achievements in the national geographic conditions monitoring project. At present, the main issue of the production of the land cover data is about how to improve the classification accuracy. For the land cover data quality inspection and acceptance, classification accuracy is also an important check point. So far, the classification accuracy inspection is mainly based on human-computer interaction or manual inspection in the project, which are time consuming and laborious. By harnessing the automatic highresolution remote sensing image change detection technology based on the ERDAS IMAGINE platform, this paper carried out the classification accuracy inspection test of land cover data in the project, and presented a corresponding technical route, which includes data pre-processing, change detection, result output and information extraction. The result of the quality inspection test shows the effectiveness of the technical route, which can meet the inspection needs for the two typical errors, that is, missing and incorrect update error, and effectively reduces the work intensity of human-computer interaction inspection for quality inspectors, and also provides a technical reference for the data production and quality control of the land cover data.
\end{abstract}

\section{INTRODUCTION}

The land cover data in the national geographic conditions monitoring project is an innovative achievement with special production technical requirements and quality characteristics, when compared with the basic surveying and mapping product. There are also some special requirements for its quality inspection content and method. At present, the most common error that has been detected in the land cover data quality inspection is the classification accuracy error, prominently reflected as missing update error and incorrect update error. The classification accuracy is the key and difficult point in the inspection and acceptance process, which need to be more focused. In the current technical conditions, there are two common methods of classification accuracy inspection, i.e., the comprehensive application of internal inspection method and reference data comparison method. The field measurement method will also be carried out by inspectors when necessary. In actual inspection work, the main check mode of inspection includes human-computer interaction and manual inspection, which are both time-consuming and laborious. Therefore, it is necessary to study a new quality inspection method using highresolution remote sensing image automatic change detection technology to improve the efficiency and accuracy of humancomputer interaction inspection.

ERDAS IMAGINE is currently a widely used remote sensing data processing software. DeltaCue is the intelligent change detection module for high resolution remote sensing image in ERDAS IMAGINE software version 2015, provides a complete set of process oriented processing solutions for high-resolution remote sensing image change detection, and an efficient and fluid visual analysis environment, which can be able to output accurate change detection results to the operator. Thus, the DeltaCue can be used for the change analysis of large-scale high-resolution remote sensing images.

Ji-wei WANG (2012) has shown that Erdas was successfully applied to emergency monitoring. Jian-hua WAN (2013) used difference principal component analysis to implement change detection, and achieved wetlands update through the decision tree classification. Tao CHEN (2017) proposed an object oriented decision tree classification method to extract the land cover data in the coastal areas. In general, most of the researches are aimed at the extraction of land cover data, but how to carry out more effective quality inspection for the data, especially the inspection method of classification accuracy has not been further studied.

In summary, this paper combines the needs of the inspection for missing update error and incorrect update error in the classification accuracy of land cover data, and proposed a complete technical route for the quality inspection aided by high-resolution remote sensing image change detection technology based on the ERDAS IMAGINE platform, and carried out related quality inspection test.

\section{METHODS AND PROCESSING}

Combined with the requirements of the classification accuracy inspection of land cover data, the technical route of this paper has been set to two parts, as shown in Figure 1.

\footnotetext{
* Corresponding author
} 
First of all is image preprocessing for previous image and new image, including geometric registration and radiation normalization. Then, change detection algorithm is applied to detect changes between the two phase of images. The change detection algorithm selected the Magnitude Diff change detection algorithm based on difference operation. The formula for calculating the variation is as follows.

$$
\alpha_{i}=\frac{T_{2}-T_{1}}{\left|T_{1}\right|}+\frac{T_{2}-T_{1}}{\left|T_{2}\right|}
$$

In the formula, the $\alpha_{i}$ refers to the variation, and the $T_{i}$ refers to the pixel value of the $\mathrm{i}$ image. For the preliminary result of change detection, the change threshold can be adjusted in the visual environment provided by the DeltaCue module. The result of change detection is obtained by human-computer interaction confirmation. The detection result can be output as a change information vector data file, and then combined with the updating data of land cover for comparison. Thus, the quality inspectors can use human-computer interaction to check the classification accuracy of results, and confirm whether there is any missing update error or incorrect update error.

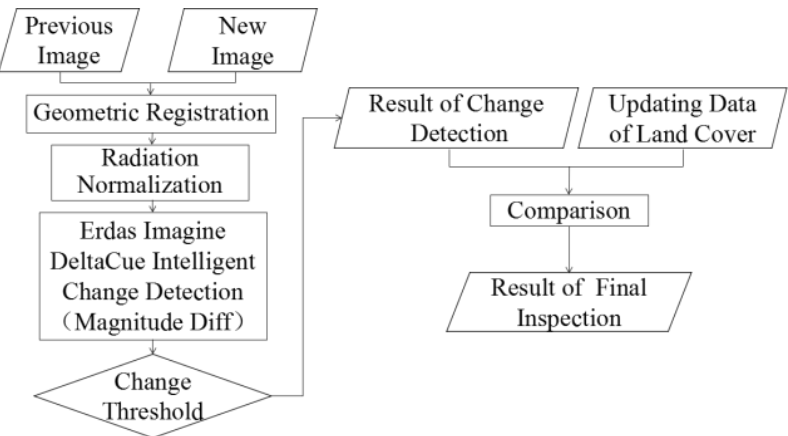

Figure 1. Technical route of classification accuracy inspection

\section{MATERIALS}

\subsection{Study Area}

The study area is located in Hetang $\left(22.71^{\circ} \mathrm{N}\right.$ to $22.73^{\circ} \mathrm{N}$ and $110.27^{\circ} \mathrm{E}$ to $110.30^{\circ} \mathrm{E}$, as shown in Figure 2), Guangxi province, covering a north-western part of the city of Beiliu of about $5.18 \mathrm{~km}^{2}$. The study area contains various types of land cover, such as road, building, water, woodland, grassland, cultivated land, construction site and so on.

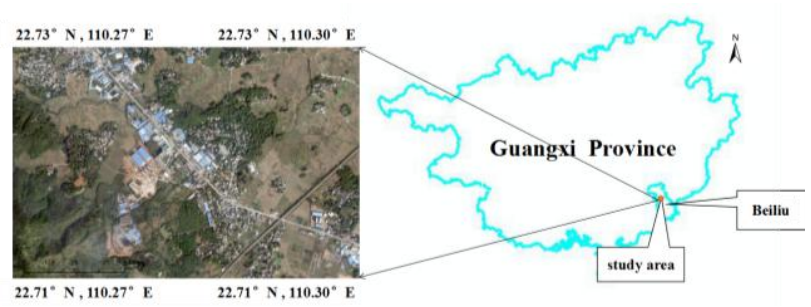

Figure 2. Study area

\subsection{Test Data}

The test data are selected from land cover data and the related image data in 2016 national geographic conditions monitoring project. The previous image is a $2015 \mathrm{ZY}-3$ satellite orthophoto with a spatial resolution of 2 meters. The new image is a 2016
GF-2 satellite orthophoto with a spatial resolution of 1 meter. The results of land cover data are mainly contains two parts, the land cover data in 2015 and the updating data of land cover in 2016 as shown in Figure 3. Based on the $2015 \mathrm{ZY}-3$ image, the GF-2 image in 2016 was conducted geometric correction, and using quadratic polynomial method and bilinear interpolation for resampling. The error is less than one meter, the result is shown in Figure 4 . The basic information of the image data used in this study is shown in Table 1.

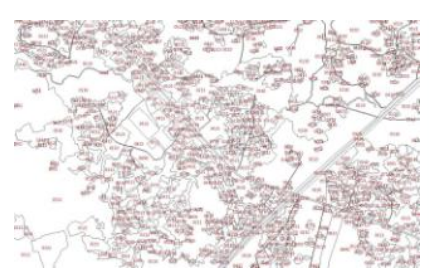

(a)

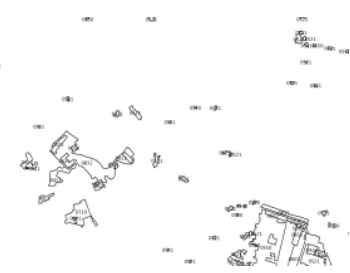

(b)
Figure 3. The results of land cover data. (a) The land cover data in 2015. (b) The updating data of land cover in 2016

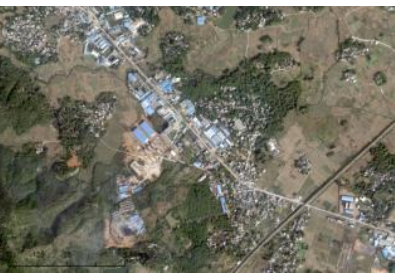

(a)

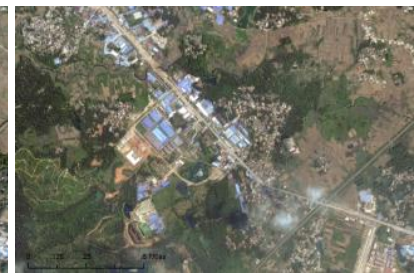

(b)
Figure 4. The satellite orthophoto of the study area. (a)April 21, 2015. (b)July 9, 2016

\begin{tabular}{|l|c|c|}
\hline Parameters & The previous image & The new image \\
\hline Satellite & ZY-3 & GF-2 \\
Acquired & $2015-04-21$ & $2016-07-09$ \\
Spatial Resolution & $2(\mathrm{~m})$ & $1(\mathrm{~m})$ \\
\hline
\end{tabular}

Table 1. The basic information of the image data used in this study

\section{RESULTS AND DISCUSSION}

The test is carried out based on the second section technical route. The results show that this method can more comprehensively detect missing update error and incorrect update error, and effectively shorten the inspection time and improve the inspection efficiency. The result of change detection for the test area is shown in Figure 5.

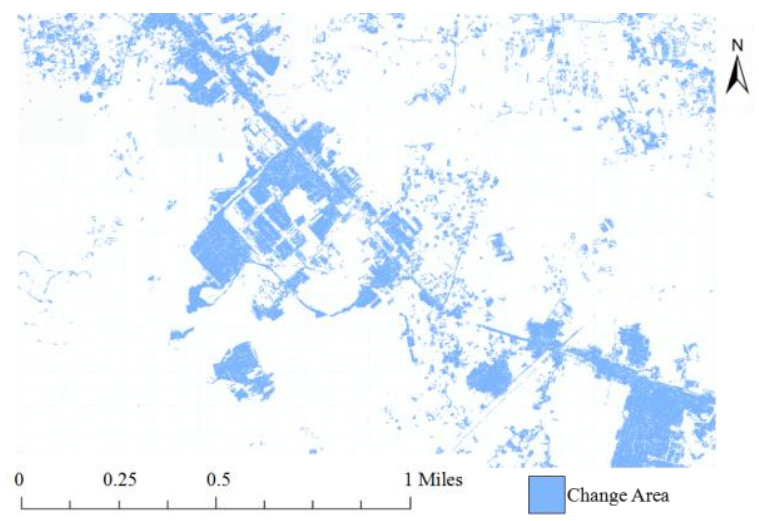

Figure 5. Result of change detection for the test area 


\subsection{Inspection for Missing Update Error}

In order to show the results more intuitively, some partial areas of the entire image were intercepted for magnification, as shown in Figure 6 and figure 7. The blue mask is the result of the change area recognition obtained by the method used in this paper and the yellow line is the updating data of land cover outlined manually by the operator during production. Now combined these two results with the 2016 GF-2 satellite orthophoto for comparison inspection, as shown in figure $6 \mathrm{c}$ and figure $7 \mathrm{c}$. It can be seen that the change detection result (i.e., blue mask) is basically the same as that of updating data of land cover (i.e., yellow line). The method given in this paper can assist quality inspectors to quickly and effectively find out missing update error. For example, as shown in the figure 6(a) and 6(b), there are some of the new-built buildings are missing update which are circled in red, and some of the new-built road is also missing update as shown in the figure 7(a) and 7(b). Obviously, this kind of missing update errors of the land cover data can be quickly found out.

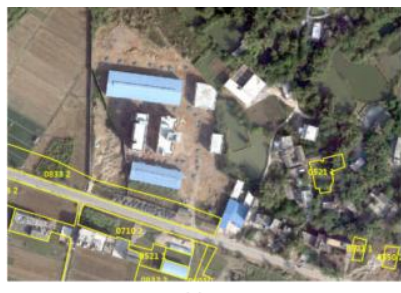

(a)

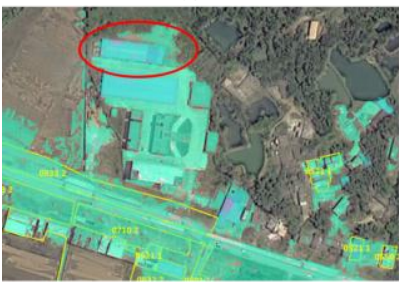

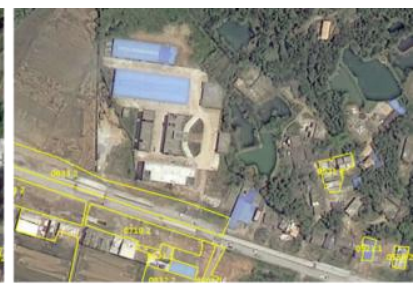

(b)

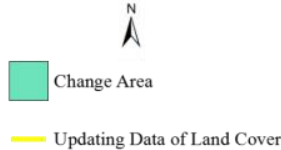

Updating Data of L and Cover

Figure 6. Missing update building inspection assisted by change detection results. (a) The previous image with updating data of land cover. (b) The new image with updating data of land cover. (c) The change detection result with updating data of land cover and the new image

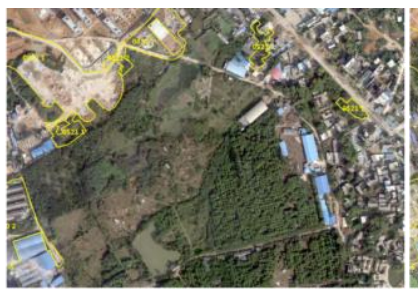

(a)

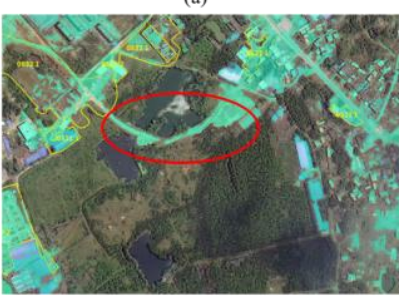

(c)

Figure 7. Missing update road inspection assisted by change detection results. (a) The previous image with updating data of land cover. (b) The new image with updating data of land cover. (c) The change detection result with updating data of land cover and the new image

\subsection{Inspection for Incorrect Update Error}

The method of this paper can also assist the quality inspector to effectively detect incorrect update error, especially the incorrect updated large polygons due to manual mistake operations. For example, as shown in figure 8 , the land cover polygon pointed by the red arrow indicates that the cultivated land (classification code is 0110) had not actually been changed, but it was incorrectly updated into buildings (classification code is 0550). And the area of this polygon is large, which is reaching $90,000 \mathrm{~m}^{2}$. This error polygon will affect the result of the image quality inspection. It can be seen that the change detection result (i.e., blue mask) obviously does not support the updating data of land cover (i.e., yellow line). Therefore, this kind of incorrect update errors of the land cover data can be quickly found out.

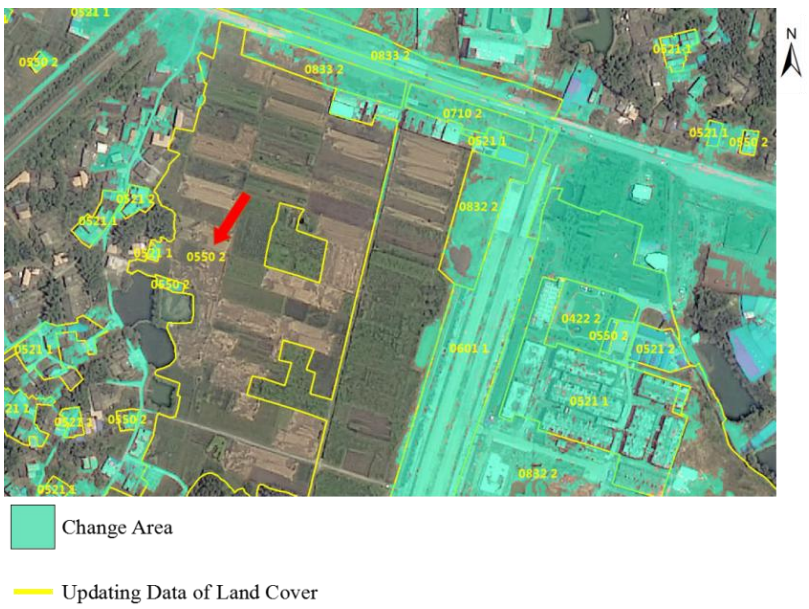

Figure 8. The inspection of incorrect update error

\subsection{Efficiency}

The test counts the time spent on inspecting the classification accuracy of land cover data. Take the study area as an example, the number of the polygons of land cover data in 2015 is 873 and the number of the polygons of the updating data in 2016 is 64. In this case, it takes twelve hours for the quality inspector to manually conduct visual inspection, and the inspection time for the method proposed in this paper is four hours, which improves the efficiency of the inspection to some extent. Moreover, there were two incorrect update errors that the manual inspection was not detected, but were found out intuitively using our method, which shows that our method can improve the accuracy of the inspection to a certain extent.

\section{CONCLUSION}

The test result indicated that change detection technology for high-resolution remote sensing images can be used to assist human-computer interaction inspection during the classification accuracy inspection of land cover data. It can effectively detect and identify errors that may exist in the land cover data, especially for the missing update error and incorrect update error. Furthermore, it can reduce the work intensity of quality inspectors, and improve the efficiency of inspection work. 
According to statistics, for the land cover data of the same area, the routine manual inspection takes twelve hours and the time using this paper method is shortened to four hours. The test also shows that if we want to apply this method effectively in the actual inspection work, the quality inspectors need to have a certain degree of professional skills. Although the DeltaCue module provides a simple and streamlined operation environment, it is still necessary for the quality inspector to master certain technical points to ensure getting the best results of change detection. Particularly, the reasonable setting of the change threshold will have a certain influence on the accuracy of the test results.

\section{ACKNOWLEDGEMENTS}

This work was support by Key Laboratory of Geo-informatics of SBSM.

\section{REFERENCES}

Wang, J.W., 2012. Application of DeltaCue in Rapid Emergency Monitoring. Science and Technology Information, 2012(2), pp. 43-44.

Zhou, M., Li, C.G., Dai, H.B., 2017. Monitoring Annual Forest Change in Eucalyptus Plantation Based on RGB-NDVI Detection of Remote Sensing Imagery. Journal of Nanjing Forestry University (Natural Sciences Edition), 41(5), pp. 65-71.

Cheng, T., Zhou, X., Liu, R.M., 2013. Land Cover Information Extraction Method Oriented to Geographic National Conditions Monitoring. Bulletin of Surveying and Mapping, 2013(8), pp. 84-86.

Zhou, X.Y., Zhang, J.X., Gao, M.X., Sang, H.Y., Zhai, L., 2017. Land Cover Information Extraction Based on High-Resolution Remote Sensing Image in Coastal Areas. Bulletin of Surveying and Mapping, 2017(2), pp. 19-24.

Fang, W.Y., Li, Y., Li, Y., 2016. Changes in Geography Census Prone Area Analysis. Geomatics and Spatial Information Technology, 33(10), pp. 147-149.

He, Z.H., Wei, Y.Q., Wang, Q., 2015. Object-oriented Extraction Method of LCA in National Conditions Monitoring. Geospatial Information, 13(2), pp. 1-3.

Wan, J.H., Li, M., Ren, G.B., Ma, Y., 2013. Efficient Method for Updating Coastal Wetland Map Based on Change Detection Technology. Remote Sensing for Land and Resources, 25(4), pp. 85-90.

CHEN, J., CHEN j., GONG P., et al, 2011. Higher Resolution Global Land Cover Mapping. Geomatics World, 2011(2), pp. 12-14.

LI, W.S., 2013. The Geographic Conditions Monitoring Promoting the Transformation and Upgrading of Surveying, Mapping and Geoinformation Industry. Geomatics World, 20(5), pp. 11-14.

LIU, J.Y., PENG, S., CHEN, J., et al, 2015. Knowledge Based Quality Checking Method and Engineering Practice of GlobeLand30 Cropland Data. Bulletin of Surveying and Mapping, 2015(4), pp. 42-48.
ZHAI, L., ZHANG X.H., SANG H.Y., et al, 2014. Land Cover Classification for National Geographic Conditions Census. Remote Sensing Information, 29(4), pp.71-74. 